\title{
MAINTAINING A DIRECTED, TRIANGULAR FORMATION OF MOBILE AUTONOMOUS AGENTS*
}

\author{
M. $\mathrm{CAO}^{\dagger}$, A. S. MORSE ${ }^{\ddagger}, \mathrm{C} \cdot \mathrm{YU}^{\S}$, B. D. O. ANDERSON§, AND S. DASGUPTA
}

\begin{abstract}
This paper analyzes a class of distributed control laws which encompasses and generalizes three previously considered types of control laws for maintaining a triangular formation in the plane consisting of three point-modeled, mobile autonomous agents. It is shown that the control laws considered can cause any initially non-collinear, positively-oriented \{resp. negatively-oriented three agent formation to converge exponentially fast to a desired positively-oriented \{resp. negativelyoriented $\}$ triangular formation. These findings extend earlier results and provide an alternative perspective.
\end{abstract}

1. Introduction. Ever since the appearance of the important work of Baillieul and Suri [2] which emphasizes the potential problem of controlling a group of mobile autonomous agents in a "directed" formation containing a cycle, interest has focused on understanding this issue in depth. A formation is directed if each agent $i$ can sense only the relative position of its "co-leaders" where by an agent $i$ 's co-leaders are meant other designated agents in the formation whose distances from agent $i$ it is the responsibility of agent $i$ to maintain. Since a directed cyclic triangular formation in the plane is the simplest formation with asymmetric co-leader relations which is both rigid and contains a cycle, it is natural to consider the problem of trying to maintain a directed triangular formation. Prompted by this, we consider the problem of maintaining a directed formation of three agents in a triangle by having each agent locally control its own position so that the distance to its co-leader for next agent in the triangle $\}$ is constant. This particular problem has recently been addressed in [3], [4] and [5]. A distinguishing feature of this paper is that it considers a class of control laws which encompasses those considered in these three earlier reference. Another distinguishing feature is that the paper explicitly takes into account in the analysis the fact that the control laws considered in [3] and [4] produce control signals which

${ }^{*}$ Dedicated to John Baillieul on the Occasion of His 65th Birthday. A preliminary version of this work was presented at the 2008 IFAC Congress [1]. The research of Cao and Morse was supported in part, by grants from the Army Research Office, the Air Force Office of Scientific Research, and the National Science Foundation and by a gift from the Xerox Corporation. The research of Yu and Anderson was supported by National ICT Australia, which is funded by the Australian Government's Department of Communications, Information Technology and the Arts and the Australian Research Council through the Backing Australia's Ability initiative and the ICT Centre of Excellence Program. The research of Dasgupta was supported by the National Science Foundation under grants ECS0225530 and ECS-0622017.

$\dagger$ University of Groningen

$\ddagger$ Yale University

$\S$ Australian National University

ฯUniversity of Iowa 
grow without bound as the points in the formation approach each other. To deal with the corresponding manifold $\mathcal{Z}$ on which the control laws are not well-defined, one must consider a dynamical system whose state space excludes $\mathcal{Z}$. We prove that unique solutions to the systems of nonlinear differential equations involved either approach $\mathcal{Z}$ or exist for all time. We explicitly characterize the closed manifold $\mathcal{N}$ on which agents are collinearly positioned and then note that $\mathcal{N}$ must contain $\mathcal{Z}$. Our main result is to show that the controls we consider will cause any initially non-collinear, "positively-oriented" \{resp. negatively-oriented $\}$ triangular formation to converge exponentially fast to a prescribed positively-oriented \{resp. negativelyoriented\} triangular formation and then come to rest. The analysis in this paper clarifies and more completely explains the results in [3] and [4]. We refer the reader to these papers for additional background and references on controlling triangular formations.

2. Triangle Formation. We consider a formation in the plane consisting of three mobile autonomous agents labeled 1,2,3 where agent 1 follows 2, 2 follows 3 and 3 follows 1 . For $i \in\{1,2,3\}$, we write $[i]$ for the label of agent $i$ 's co-leader where $[1]=2,[2]=3$ and $[3]=1$. We assume that the desired distance between agents $i$

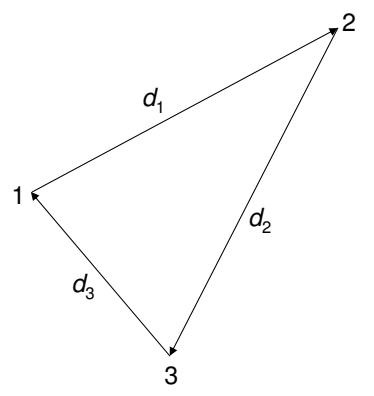

Fig. 1. Directed Point Formation

and $[i]$ is $d_{i}$; here the $d_{i}$ are positive numbers which satisfy the triangle inequalities:

$$
d_{1}<d_{2}+d_{3} \quad d_{2}<d_{1}+d_{3} \quad d_{3}<d_{1}+d_{2}
$$

Note that there are two distinct triangular formations which satisfy the desired distance constraints. The first is as shown in Figure 1 and is referred to as a clockwiseoriented triangle. The second, called a counterclockwise oriented triangle is the triangle which results when the triangle shown in Figure 1 is flipped over.

In the sequel we write $x_{i}$ for the Cartesian coordinate vector of agent $i$ in some fixed global coordinate system in the plane, and $y_{i j}$ for the position of agent $j$ in some fixed coordinate system of agent $i$ 's choosing. Thus for $i \in\{1,2,3\}$, there is a rotation matrix $R_{i}$ and a translation vector $\tau_{i}$ such that $y_{i j}=R_{i} x_{j}+\tau_{i}, j \in\{1,2,3\}$. We assume that agent $i$ 's motion is described by a simple kinematic point model of 
the form

$$
\dot{y}_{i i}=u_{i} \quad i \in\{1,2,3\}
$$

where $u_{i}$ is agent $i$ 's control input. Thus in global coordinates,

$$
\dot{x}_{i}=R_{i}^{-1} u_{i}, \quad i \in\{1,2,3\}
$$

We assume that for $i \in\{1,2,3\}$, agent $i$ can measure the relative position of agent $[i]$ in its own coordinate system. This means that for $i \in\{1,2,3\}$, agent $i$ can measure the signal $R_{i} z_{i}$ where

$$
z_{i}=x_{i}-x_{[i]}, \quad i \in\{1,2,3\}
$$

Our aim is to define control laws of a sufficiently general form to encompass the control laws studied previously in $[5,4,3]$. Towards this end we consider controls of the form

$$
u_{i}=-R_{i} z_{i} e_{i}, \quad i \in\{1,2,3\}
$$

where

$$
e_{i}=g_{i}\left(\left\|R_{i} z_{i}\right\|^{2}-d_{i}^{2}\right), \quad i \in\{1,2,3\}
$$

and $g_{i}$ is any given strictly monotone increasing function which is defined and continuously differentiable on the open interval $\left(-d_{i}^{2}, \infty\right)$ and satisfies $g_{i}(0)=0$. Thus $u_{i}$ is a well-defined, continuously differentiable control law on open space

$$
\mathbb{R}^{6}-\mathcal{Z}_{i}
$$

where $\mathcal{Z}_{i}=\left\{x: z_{i}=0\right\}$ and $\mathbb{R}^{6}-\mathcal{Z}_{i}$ is the complement of $\mathcal{Z}_{i}$ in $\mathbb{R}^{6}$. Note that the rotation matrices do not affect the definition of the $e_{i}$ in that

$$
e_{i}=g_{i}\left(\left\|z_{i}\right\|^{2}-d_{i}^{2}\right), \quad i \in\{1,2,3\}
$$

Moreover $R_{i}$ cancels out of the update equation

$$
\dot{x}_{i}=-z_{i} e_{i}, \quad i \in\{1,2,3\}
$$

Set

$$
\mathcal{Z}=\mathcal{Z}_{1} \cup \mathcal{Z}_{2} \cup \mathcal{Z}_{3}
$$

The closed loop system of interest is thus the smooth, time-invariant, dynamical system on the state space

$$
\mathcal{X}=\mathbb{R}^{6}-\mathcal{Z}
$$


described in global coordinates by the equations

$$
\dot{x}_{i}=-\left(x_{i}-x_{[i]}\right) g_{i}\left(\left\|x_{i}-x_{[i]}\right\|^{2}-d_{i}^{2}\right), \quad i \in\{1,2,3\}
$$

In the sequel we shall refer this system as the overall system.

Examples: The simplest type of controls which satisfies the assumptions on the $g_{i}$, are control laws in which the $e_{i}$ are defined by

$$
e_{i}=\left\|R_{i} z_{i}\right\|^{2}-d_{i}^{2}, \quad i \in\{1,2,3\}
$$

In this case the $g_{i}$ are the function $g_{i}:\left(-d_{i}^{2}, \infty\right) \rightarrow \mathbb{R}, w \longmapsto g_{i}(w)=w, \quad i \in\{1,2,3\}$. Note that in this case there is no problem in extending the domain of definition of the $g_{i}$ to all of $\mathbb{R}$. Doing this enables one to take all of $\mathbb{R}^{6}$ as the state space of (8) as was done in [5].

The controls considered in [3] use "normalized" errors of the form

$$
e_{i}=\frac{1}{\left\|R_{i} z_{i}\right\|^{2}}\left(\left\|R_{i} z_{i}\right\|^{2}-d_{i}^{2}\right), \quad i \in\{1,2,3\}
$$

In this case the $g_{i}$ are the functions

$$
g_{i}(w)=1-\frac{d_{i}^{2}}{\left(w+d_{i}^{2}\right)}, \quad i \in\{1,2,3\}
$$

The controls considered in [4] use the errors

$$
e_{i}=\frac{1}{\left\|R_{i} z_{i}\right\|}\left(\left\|R_{i} z_{i}\right\|-d_{i}\right), \quad i \in\{1,2,3\}
$$

In this case the $g_{i}$ are

$$
g_{i}(w)=1-\frac{d_{i}}{\left(w+d_{i}^{2}\right)^{\frac{1}{2}}}, \quad i \in\{1,2,3\}
$$

In both cases the $g_{i}$ are strictly monotone increasing functions which are defined and continuously differentiable on $\left(-d_{i}^{2}, \infty\right)$.

3. Analysis. Our aim is to study the geometry of the overall system defined by (8). Towards this end let

$$
e=\left[\begin{array}{l}
e_{1} \\
e_{2} \\
e_{3}
\end{array}\right] \quad x=\left[\begin{array}{l}
x_{1} \\
x_{2} \\
x_{3}
\end{array}\right] \quad z=\left[\begin{array}{c}
z_{1} \\
z_{2} \\
z_{3}
\end{array}\right]
$$

Note at once that because of Lipschitz continuity, for any initial state $x(0)=y \in \mathcal{X}$ there must exist a largest interval $\left[0, T_{y}\right)$ on which a unique solution to (8) exists. Next note that as a consequence of the definitions of the $z_{i}$ in (3),

$$
z_{1}+z_{2}+z_{3}=0
$$


and

$$
\dot{z}_{i}=-z_{i} e_{i}+z_{[i]} e_{[i]}, \quad i \in\{1,2,3\}
$$

Observe that the equilibrium points of the overall system are those values of the $x_{i}$ for which

$$
z_{i} e_{i}=0, \quad i \in\{1,2,3\}
$$

Since $z_{i} \neq 0$ for $x \in \mathcal{X}$

$$
\mathcal{E}=\{x: e=0, x \in \mathcal{X}\}
$$

is the equilibrium set of the overall system. It is possible to show by example that this set is not globally attractive and thus that the overall system is not globally asymptotically stable. On the other hand it will be shown that there is a thin set in $\mathbb{R}^{6}$ outside of which all trajectories approach $\mathcal{E}$ exponentially fast. The set to which we are referring corresponds to those formations in $\mathcal{X}$ which are collinear. To explicitly characterize this set, we need the following fact.

LEMma 1. The points at $x_{1}, x_{2}, x_{3}$ in $\mathbb{R}^{6}$ are collinear if and only if $\operatorname{rank}\left[\begin{array}{lll}z_{1} & z_{2} & z_{3}\end{array}\right]<2$

The simple proof is omitted.

To proceed, let $\mathcal{N}$ denote the set of points in $\mathbb{R}^{6}$ corresponding to points in the plane which are collinear. In other words

$$
\mathcal{N}=\left\{x: \operatorname{rank}\left[\begin{array}{lll}
z_{1} & z_{2} & z_{3}
\end{array}\right]<2, z_{1}+z_{2}+z_{3}=0\right\}
$$

Note that $\mathcal{N}$ is a closed manifold in $\mathbb{R}^{6}$. Note in addition that $\mathcal{N}$ contains $\mathcal{Z}$ and is small enough to not intersect $\mathcal{E}$ :

LEMma 2. $\mathcal{N}$ and $\mathcal{E}$ are disjoint sets.

Proof. To show that $\mathcal{N} \cap \mathcal{E}$ is empty, we assume the contrary. Let $x \in \mathcal{N} \cap \mathcal{E}$ be fixed. Since $\mathcal{E}$ and $\mathcal{Z}$ are disjoint, $x \notin \mathcal{Z}$. Therefore $z_{i} \neq 0$ for some $i \in\{1,2,3\}$. Then there must be a number $\lambda$ such that $z_{[i]}=\lambda z_{i}$. Hence $z_{[i]+1}=-(1+\lambda) z_{i}$. But $x \in \mathcal{E}$ so $\left\|z_{i}\right\|=d_{i}, \quad i \in\{1,2,3\}$. Thus $|\lambda| d_{i}=d_{[i]}$ and $|1+\lambda| d_{i}=d_{[i]+1}$. Then $d_{i}+d_{[i]}=d_{[i]+1}$ when $\lambda \geq 0, d_{i}+d_{[i]+1}=d_{[i]}$ when $\lambda \leq-1$, and $d_{[i]}+d_{[i]+1}=d_{i}$ when $-1<\lambda<0$. All of these equalities contradict (1). Therefore $\mathcal{N}$ and $\mathcal{E}$ are disjoint sets.

That $\mathcal{N} \cap \mathcal{X}$ might be the place where formation control will fail is further underscored by the fact that formation's points in $\mathcal{X}$ which are initially collinear, remain collinear along all trajectories starting at such points. To understand why this is so, first note that for any two vectors $p, q \in \mathbb{R}^{2}, \operatorname{det}\left[\begin{array}{ll}p & q\end{array}\right]=p^{\prime} G q$ where

$$
G=\left[\begin{array}{cc}
0 & 1 \\
-1 & 0
\end{array}\right]
$$


From this and (13) it follows that $\operatorname{det}\left[z_{1} \quad z_{2}\right]=-\operatorname{det}\left[\begin{array}{ll}z_{1} & z_{3}\end{array}\right]$. This and the definition of $\mathcal{N}$ in (17) imply that

$$
\mathcal{N} \cap \mathcal{X}=\left\{x: x \in \mathcal{X}, \operatorname{det}\left[z_{1} \quad z_{2}\right]=0\right\}
$$

Moreover (14) implies that

$$
\frac{d}{d t} \operatorname{det}\left[\begin{array}{ll}
z_{1} & z_{2}
\end{array}\right]=-\left(e_{1}+e_{2}+e_{3}\right) \operatorname{det}\left[\begin{array}{ll}
z_{1} & z_{2}
\end{array}\right]
$$

Thus if $\operatorname{det}\left[z_{1} \quad z_{2}\right]=0$ at $t=0$, then $\operatorname{det}\left[\begin{array}{ll}z_{1} & z_{2}\end{array}\right]=0$ along all the trajectory of the overall system starting at $y$.

It can be shown that there are initially collinear formations in $\mathcal{N} \cap \mathcal{X}$ which tend to the boundary of $\mathcal{X}$, which of course is a form of instability. Despite the fact that misbehavior can occur within $\mathcal{N} \cap \mathcal{X}$, the dimension of $\mathcal{N}$ is less than 6 which means that "almost every" initial formation in $\mathcal{X}$ will be non-collinear. The good news is that all such initially non-collinear formations will converge to the desired formation and come to rest, and moreover, the convergence will occur exponentially fast. This is in essence, the geometric interpretation of our main result on triangular formations.

THEOREM 1. Each trajectory of the overall system (8) starting outside of $\mathcal{N}$, converges exponentially fast to a finite limit point in $\mathcal{E}$.

The set of points $\mathcal{X}-\mathcal{N} \cap \mathcal{X}$ consists of two disjoint point sets, one for which $\operatorname{det}\left[\begin{array}{ll}z_{1} & z_{2}\end{array}\right]>0$ and the other for which $\operatorname{det}\left[z_{1} \quad z_{2}\right]<0$. Once the theorem has been proved, it is easy to verify that formations starting at points such that $\operatorname{det}\left[\begin{array}{ll}z_{1} & z_{2}\end{array}\right]<0$, converge to the clockwise oriented triangular formation shown in Figure 1 whereas formations starting at points such that $\operatorname{det}\left[\begin{array}{ll}z_{1} & z_{2}\end{array}\right]>0$, converge to the corresponding counterclockwise oriented triangular formation.

The proof of Theorem 1 involves several steps. The first is to show that all trajectories in $\mathcal{X}$ which do not tend to $\mathcal{Z}$, exist for all time. To accomplish this, let $\Omega:\left(-d_{1}^{2}, \infty\right) \times\left(-d_{2}^{2}, \infty\right) \times\left(-d_{3}^{2}, \infty\right) \rightarrow \mathbb{R}$ denote the function

$$
\Omega\left(w_{1}, w_{2}, w_{3}\right)=\int_{0}^{w_{1}} g_{1}(s) d s+\int_{0}^{w_{2}} g_{2}(s) d s+\int_{0}^{w_{3}} g_{3}(s) d s
$$

Observe that the constraints on the $g_{i}$ imply that $\Omega$ is continuously differentiable and positive definite; moreover $\Omega$ is an unbounded function of $w$ where $w=\left[\begin{array}{lll}w_{1} & w_{2} & w_{3}\end{array}\right]^{\prime}$. Let

$$
V=\Omega\left(\left\|z_{1}\right\|^{2}-d_{1}^{2},\left\|z_{2}\right\|^{2}-d_{2}^{2},\left\|z_{3}\right\|^{2}-d_{3}^{2}\right)
$$

Fix $y \in \mathcal{X}$ and let $\left[0, T_{y}\right)$ denote the maximal interval of existence of the overall system. Then for $t \in\left[0, T_{y}\right)$,

$$
\begin{gathered}
\dot{V}=-2\left\{\left(z_{1}^{\prime} z_{1} e_{1}^{2}-z_{1}^{\prime} z_{2} e_{1} e_{2}\right)+\left(z_{2}^{\prime} z_{2} e_{2}^{2}-z_{2}^{\prime} z_{3} e_{2} e_{3}\right)\right. \\
\left.+\left(z_{3}^{\prime} z_{3} e_{3}^{2}-z_{3}^{\prime} z_{1} e_{3} e_{1}\right)\right\}
\end{gathered}
$$


or

$$
\dot{V}=-\left\|z_{1} e_{1}-z_{2} e_{2}\right\|^{2}-\left\|z_{2} e_{2}-z_{3} e_{3}\right\|^{2}-\left\|z_{3} e_{3}-z_{1} e_{1}\right\|^{2}
$$

Thus $V$ is monotone non-increasing on $\left[0, T_{y}\right)$. Since $V$ is also bounded below by 0 , $V$ must be bounded on $\left[0, T_{y}\right)$. In view of the fact that $\Omega$ is a continuous, unbounded function of $w$, each $\left\|z_{i}\right\|^{2}-d_{i}^{2}$ is also bounded on $\left[0, T_{y}\right)$. Therefore each $z_{i}$ is bounded on $\left[0, T_{y}\right)$. In view of $(8), \dot{x}$ is also bounded on $\left[0, T_{y}\right)$. At this point one of two things can happen: Either $x \rightarrow \mathcal{Z}$ as $t \rightarrow T_{y}$ or it does not. We are interested in the latter situation in which case either $T_{y}=\infty$ or $T_{y}<\infty$. If it were true that $T_{y}<\infty$, then $x$ would approaches the finite limit

$$
\bar{x}=\int_{0}^{T_{y}} \dot{x} d t
$$

as $t \rightarrow T_{y}$. But if this were so, then there would have to be an interval $\left[T_{y}, T^{\prime}\right)$ of positive length on which a solution to the overall system starting at $\bar{x}$ exists. Uniqueness would then imply the existence on $\left[0, T^{\prime}\right)$ of a solution to the overall system starting at $y$. This contradicts the assumption that $\left[0, T_{y}\right)$ is the interval of maximal existence. Thus $T_{y}=\infty$. We summarize.

Proposition 1. Each trajectory of the overall system either tends to $\mathcal{Z}$ or exists on $[0, \infty)$. Moreover $z$ is bounded along any trajectory of the overall system which does not approach $\mathcal{Z}$.

More can be said if $y \notin \mathcal{N}$. Suppose that this is so in which case $\operatorname{det}\left[z_{1}(0) z_{2}(0)\right] \neq$ 0 because of (17). Suppose $T_{y}<\infty$. In view of Proposition 1, $x$ would tend to $\mathcal{Z}$ as $t \rightarrow T_{y}$. Since $\mathcal{Z} \subset \mathcal{N}, x$ would therefore tend to $\mathcal{N}$ as $t \rightarrow T_{y}$. In other words, $\operatorname{det}\left[\begin{array}{ll}z_{1} & z_{2}\end{array}\right] \rightarrow 0$ as $t \rightarrow T_{y}$, again because of (17). But this is impossible because of (19). Therefore $T_{y}=\infty$. We've proved the following.

COROLlary 1. Each trajectory of the overall system which starts outside of $\mathcal{N}$ exists on $[0, \infty)$ and remains outside of $\mathcal{N}$ for $t<\infty$.

Our next goal is to show that there is an open set of points in $\mathcal{X}$ from which all solutions to the overall system tend to $\mathcal{E}$ exponentially fast. For this we will need the following.

Lemma 3. Let $\rho$ be a positive number. There exist positive numbers $\mu$ and $\delta$ such that

$$
\begin{aligned}
& \Omega\left(w_{1}, w_{2}, w_{3}\right) \geq \frac{\mu}{2}\|w\|^{2}, \quad\|w\|^{2} \leq \rho \\
& \Omega\left(w_{1}, w_{2}, w_{3}\right) \leq \frac{\delta}{2}\|w\|^{2}, \quad\|w\|^{2} \leq \rho
\end{aligned}
$$

Proof. Let

$$
\mu_{i}=\inf _{|s| \leq \sqrt{\rho}} \frac{d g_{i}(s)}{d s}, i \in\{1,2,3\}
$$


Each $\mu_{i}$ is positive because each $g_{i}$ is continuously differentiable and strictly increasing. From this and the assumption that $g_{i}(0)=0, i \in\{1,2,3\}$ it follows that

$$
\left|g_{i}(s)\right| \geq \mu_{i}|s|,|s| \leq \sqrt{\rho}, i \in\{1,2,3\}
$$

Therefore

$$
\int_{0}^{w_{i}} g_{i}(s) d s \geq \frac{\mu_{i}}{2} w_{i}^{2}, \quad\left|w_{i}\right| \leq \sqrt{\rho}
$$

Set $\mu=\min \left\{\mu_{1}, \mu_{2}, \mu_{3}\right\}$. It follows that (21) is true.

Since each $g_{i}$ is Lipschitz continuous and satisfies $g_{i}(0)=0$, there are positive constants $\delta_{i}, i \in\{1,2,3\}$ such that $\left|g_{i}(s)\right| \leq \delta_{i}|s|,|s| \leq \sqrt{\rho}$. It follows from this that

$$
\int_{0}^{w_{i}} g_{i}(s) d s \leq \frac{\delta_{i}}{2} w_{i}^{2}, \quad\left|w_{i}\right| \leq \sqrt{\rho}
$$

Set $\delta=\max \left\{\delta_{1}, \delta_{2}, \delta_{3}\right\}$. It follows that $(22)$ is true.

To proceed, observe from (20) that

$$
\dot{V}=-e^{\prime} Q^{\prime} Q e
$$

where

$$
Q=\left[\begin{array}{ccc}
-z_{1} & z_{2} & 0 \\
0 & -z_{2} & z_{3} \\
z_{1} & 0 & -z_{3}
\end{array}\right]
$$

Note that $Q$ is also the transpose of the rigidity matrix $\{[6]\}$ of the point formation shown in Figure 1. By inspection it is clear that the rank of $Q$ is less than three just in case, for at least one distinct pair of integers $i, j \in\{1,2,3\}, z_{i}$ is a scalar multiple of $z_{j}$; moreover because of (13), for such $i$ and $j z_{k}$ would also have to be a scalar multiple of $z_{j}$ where $k$ is the remaining integer in $\{1,2,3\}$. In other words,

$$
\operatorname{rank} Q<3 \Longleftrightarrow \operatorname{rank}\left[\begin{array}{lll}
z_{1} & z_{2} & z_{3}
\end{array}\right]<2
$$

In the light of this and the definition of $\mathcal{N}$, it is clear that $Q^{\prime} Q$ is positive definite if and only if $x \notin \mathcal{N}$. For any positive number $\eta$, define

$$
\mathcal{S}(\eta)=\left\{x: \sum_{i=1}^{3}\left(\left\|z_{i}\right\|^{2}-d_{i}^{2}\right)^{2}<\eta, \quad z_{1}+z_{2}+z_{3}=0, x \in \mathcal{X}\right\}
$$

Note that for any $\eta>0, \mathcal{E} \subset \mathcal{S}(\eta)$ and $\mathcal{S}(\eta) \rightarrow \mathcal{E}$ as $\rho \rightarrow 0$. In view of Lemma 2 it is possible to choose $\eta>0$ so small that $\mathcal{N}$ and $\mathcal{S}(\eta)$ are disjoint. Pick $\rho>0$ so that this is so and also so that $\rho<\min \left\{d_{1}^{2}, d_{2}^{2}, d_{3}^{2}\right\}$. This last inequality ensures that the closure of $\mathcal{S}(\rho)$ and $\mathcal{Z}$ are also disjoint.

To proceed, let $\mu$ and $\delta$ be as in Lemma 3 and note from the inequalities therein that $\mu \leq \delta$. Pick any positive number $\rho^{*}<\frac{\mu}{\delta} \rho$. Since $\frac{\mu}{\delta} \leq 1, \mathcal{S}\left(\rho^{*}\right)$ is a strictly proper 
subset of $\mathcal{S}(\rho)$. It will now be shown that any trajectory starting in $\mathcal{S}\left(\rho^{*}\right)$ remains in $\mathcal{S}(\rho)$ and converges to $\mathcal{E}$ exponentially fast. Towards this end fix $y \in \mathcal{S}\left(\rho^{*}\right)$. Since $\mathcal{S}\left(\rho^{*}\right)$ and $\mathcal{N}$ are disjoint, $T_{y}=\infty$ because of Corollary 1. Let $\{x(t): t \in[0, \infty)\}$ denote the corresponding trajectory of the overall system starting at $y$. Since $x(0) \in$ $\mathcal{S}\left(\rho^{*}\right)$ and $\mathcal{S}\left(\rho^{*}\right)$ is a strictly proper subset of $\mathcal{S}(\rho)$, there must be a positive time $T^{\prime}$ such that $x(t) \in \mathcal{S}(\rho), t \in\left[0, T^{\prime}\right)$. Let $T^{*}$ be the largest such time. In view of (22), and the definition of $V$,

$$
V \leq \frac{\delta}{2} \sum_{i=1}^{3}\left(\left\|z_{i}\right\|^{2}-d_{i}^{2}\right)^{2}, t \in\left[0, T^{*}\right)
$$

But $x(0) \in \mathcal{S}\left(\rho^{*}\right)$ so

$$
\sum_{i=1}^{3}\left(\left\|z_{i}(0)\right\|^{2}-d_{i}^{2}\right)^{2}<\rho^{*}
$$

From this, (24) and the fact that $V$ is non-decreasing on $\left[0, T^{*}\right)$, there follows $V(t)<$ $\frac{\delta}{2} \rho^{*}, \quad t \in\left[0, T^{*}\right)$. But in view of $(21)$, and the definition of $V$,

$$
V \geq \frac{\mu}{2} \sum_{i=1}^{3}\left(\left\|z_{i}\right\|^{2}-d_{i}^{2}\right)^{2}, \quad t \in\left[0, T^{*}\right)
$$

Therefore

$$
\sum_{i=1}^{3}\left(\left\|z_{i}\right\|^{2}-d_{i}^{2}\right)^{2}<\frac{\delta}{\mu} \rho^{*}, t \in\left[0, T^{*}\right)
$$

Suppose $T^{*}<\infty$. Then (26) implies that

$$
\sum_{i=1}^{3}\left(\left\|z_{i}\right\|^{2}-d_{i}^{2}\right)^{2} \leq \frac{\delta}{\mu} \rho^{*}, t \in\left[0, T^{*}\right]
$$

But $\frac{\delta}{\mu} \rho^{*}<\rho$, so

$$
\sum_{i=1}^{3}\left(\left\|z_{i}\right\|^{2}-d_{i}^{2}\right)^{2}<\rho, t \in\left[0, T^{*}\right]
$$

Because of continuity, this means there is an interval $\left[0, T^{\prime}\right)$ larger than $\left[0, T^{*}\right)$ such that $x(t) \in \mathcal{S}(\rho), t \in\left[0, T^{\prime}\right)$. This is impossible because $\left[0, T^{*}\right)$ was defined to be the largest such interval. Therefore $T^{*}=\infty$. This proves that the trajectory remains in $\mathcal{S}(\rho)$ for all time.

It will now be shown that $x$ tends to $\mathcal{E}$ exponentially fast. For this let $\widehat{\mathcal{S}}$ denote the closure of $\{z: x \in \mathcal{S}(\rho)\}$. It is clear that $\widehat{\mathcal{S}}$ is compact. In addition, since the closure of $\mathcal{S}(\rho)$ and $\mathcal{N}$ are disjoint, $\pi\left(Q^{\prime} Q\right)>0, z \in \widehat{\mathcal{S}}$, where $\pi\left(Q^{\prime} Q\right)$ is the smallest eigenvalue of $Q^{\prime} Q$. Thus if we define

$$
\lambda=\inf _{z \in \widehat{\mathcal{S}}} \pi\left(Q^{\prime} Q\right)
$$


then $\lambda>0$ and for $t \in[0, \infty)$

$$
\dot{V} \leq-\lambda\|e\|^{2}
$$

But $e_{i}=g\left(\left\|z_{i}\right\|^{2}-d_{i}^{2}\right), i \in\{1,2,3\}$. In view of (23) and the fact that $x(t) \in \mathcal{S}(\rho)$ for all time, $\left|e_{i}\right| \geq \mu_{i}||\left|z_{i} \|^{2}-d_{i}^{2}\right|, i \in\{1,2,3\}$. This implies that

$$
\|e\|^{2} \geq \mu^{2} \sum_{i=1}^{3}\left(\left\|z_{i}\right\|^{2}-d_{i}^{2}\right)^{2}
$$

where $\mu=\min \left\{\mu_{1}, \mu_{2}, \mu_{3}\right\}$. From this and (24) there follows $\|e\|^{2} \geq \frac{2}{\delta} \mu^{2} V$. Combining this with $(28)$ one gets

$$
\dot{V} \leq-\frac{2}{\delta} \lambda \mu^{2} V
$$

Therefore by the Bellman-Gronwall Lemma

$$
V \leq V(0) e^{-\frac{2}{\delta} \lambda \mu^{2} t}, \quad t \geq 0
$$

so $V$ tends to 0 exponentially fast. In view of $(25)$, each $\left(\left\|z_{i}\right\|^{2}-d_{i}^{2}\right), i \in\{1,2,3\}$ also tends to zero exponentially fast. This proves that the trajectory under consideration approaches $\mathcal{E}$ exponentially fact. We summarize:

Proposition 2. There exists an open set of points in $\mathcal{X}$, namely $\mathcal{S}\left(\rho^{*}\right)$, such that all trajectories of the overall system starting in $\mathcal{S}\left(\rho^{*}\right)$, converge to $\mathcal{E}$ exponentially fast.

Note that for a trajectory to converge to $\mathcal{E}$ means that the corresponding formation converges to the desired triangle. To show that the formation actually comes to rest is a simple matter of exploiting the fact that the $\left\|\dot{x}_{i}\right\|$ are bounded above by signals which are decaying to zero exponentially fast. A proof of this last observation will not be given here.

To show that all trajectories outside of $\mathcal{N}$ converge exponentially fast to $\mathcal{E}$ requires more work. In view of Proposition 2, we already know that any trajectory which enters $\mathcal{S}\left(\rho^{*}\right)$ in finite time must converge to $\mathcal{E}$ exponentially fast. The problem then is to show that any trajectory starting outside of $\mathcal{N}$ must enter $\mathcal{S}\left(\rho^{*}\right)$ in finite time. A key observation from (20) needed to prove this is that $\dot{V}<0$ whenever the three velocity vectors $z_{i} e_{i}, i \in\{1,2,3\}$ are not all equal. Prompted by this, let

$$
\mathcal{M}=\mathcal{Z}_{0} \bigcup_{i=0}^{3} \mathcal{M}_{i}
$$

where

$$
\mathcal{Z}_{0}=\mathcal{Z}_{1} \cap \mathcal{Z}_{2} \cap \mathcal{Z}_{3}
$$




$$
\mathcal{M}_{0}=\left\{x: x \in \mathcal{N} \cap \mathcal{X}, z_{1} e_{1}=z_{2} e_{2}=z_{3} e_{3}\right\}
$$

and for $i \in\{1,2,3\}$

$$
\mathcal{M}_{i}=\left\{x: z_{i}=0, z_{[i]} e_{[i]}=z_{[i]]]} e_{[[i]]}, x \in \mathbb{R}^{6}-\mathcal{Z}_{[i]} \cup \mathcal{Z}_{[[i]]}\right\}
$$

Note that $\mathcal{M} \subset \mathcal{N}$ and that $\mathcal{M}$ and $\mathcal{E}$ are disjoint because $\mathcal{N}$ and $\mathcal{E}$ are.

To show that trajectories starting outside of $\mathcal{N}$ must converge exponentially fast to $\mathcal{E}$, it is enough to show that all such trajectories are bounded away from $\mathcal{M}$, even in the limit as $t \rightarrow \infty$. In the sequel we explain why this is so.

Consider the function $\Phi: \mathcal{X} \rightarrow \mathbb{R}$ given by $\Phi(x)=-\left\|z_{1} e_{1}-z_{2} e_{2}\right\|^{2}-\| z_{2} e_{2}-$ $z_{3} e_{3}\left\|^{2}-\right\| z_{3} e_{3}-z_{1} e_{1} \|^{2}$ with the $z_{i}$ and $e_{i}$ as defined previously. Obviously $\Phi(x(t))=\dot{V}$ when $x(t)$ is a solution to the overall system. We are interested in the following property of $\Phi$ when viewed as a function on $\mathcal{X}$.

Lemma 4. Let $\mathcal{T}$ be any subset of $\mathcal{X}$ whose closure is disjoint with $\mathcal{M} \cup \mathcal{E}$. Then

$$
\sup _{x \in \mathcal{T}} \Phi(x)<0
$$

Proof. Observe that (29) will be true if

$$
\Phi(x)<0, \quad x \in \mathcal{T}
$$

and

$$
\lim _{x \rightarrow \mathcal{B}} \Phi(x)<0
$$

where $\mathcal{B}$ is the boundary of $\mathcal{T}$. Note in addition that $\Phi(x)<0$ whenever $\Phi(x) \neq 0$. Thus (30) and (31) are equivalent to

$$
\Phi(x) \neq 0, \quad x \in \mathcal{T}
$$

and

$$
\lim _{x \rightarrow \mathcal{B}} \Phi(x) \neq 0
$$

respectively.

Since $\mathcal{T}$ and $\mathcal{M} \cup \mathcal{E}$ are disjoint, to prove (32) it is sufficient to prove that if $\Phi(x)=0$ for some $x \in \mathcal{X}$, then $x \in \mathcal{M} \cup \mathcal{E}$. Therefore suppose $\Phi(x)=0$ for some $x \in \mathcal{X}$. If $e=0$, then it is clear that $x \in \mathcal{E} \cup \mathcal{M}$. Suppose $e \neq 0$ in which case at least one $e_{i}$ is nonzero. Moreover, since $\Phi$ is well defined on $\mathcal{X}, z_{1} e_{1}=z_{2} e_{2}=z_{3} e_{3}$ because of the definition of $\Phi$. Thus the three $z_{i}$ are scalar multiples of one of them. Hence by Lemma $1, x \in \mathcal{N}$. It follows that $x$ is in $\mathcal{M}_{0}$ and consequently in $\mathcal{M} \cup \mathcal{E}$. Thus (32) is true. 
Since $\mathcal{B}$ and $\mathcal{M} \cup \mathcal{E}$ are disjoint closed sets, to prove (33) it is enough to show that if $\Phi(x) \rightarrow 0$ then $x \rightarrow \mathcal{M} \cup \mathcal{E}$. Suppose $\Phi(x) \rightarrow 0$ in which case either $x \rightarrow \mathcal{X}$ or $x \rightarrow \mathcal{Z}$ because $\mathbb{R}^{6}=\mathcal{X} \cup \mathcal{Z}$. If the former true, then clearly $x \rightarrow \mathcal{M} \cup \mathcal{E}$ because as was just proved, the relations $\Phi(x)=0$ and $x \in \mathcal{X}$ imply $x \in \mathcal{M} \cup \mathcal{E}$.

Suppose $x \rightarrow \mathcal{Z}$ in which case for at least one $i, z_{i} \rightarrow 0$. Without loss of generality, suppose $i=1$. Note that if $x \rightarrow \mathcal{Z}_{0}$, then $x \rightarrow \mathcal{M}$ because of the definition of $\mathcal{M}$. Suppose therefore that $x \nrightarrow \mathcal{Z}_{0}$. This means that for some $i$ - say $i=2-z_{2} \nrightarrow 0$. By (13), $z_{3} \nrightarrow 0$. Thus $x \rightarrow \mathbb{R}^{6}-\mathcal{Z}_{2} \cup \mathcal{Z}_{3}$. Moreover $z_{3} e_{3}-z_{2} e_{2}$ tends to zero because $\Phi(x) \rightarrow 0$. In view of the definition of $\mathcal{M}_{2}$, it is therefore clear that $x \rightarrow \mathcal{M}_{2}$. Therefore $x \rightarrow \mathcal{M} \cup \mathcal{E}$.

To proceed, suppose that $x(t)$ is a trajectory starting outside of $\mathcal{N}$ and that for all $t, x(t)$ is bounded away from $\mathcal{M}$. Then

$$
\gamma=\inf _{t \rightarrow \infty} \delta(x(t))
$$

must be a positive number where for $x \in \mathbb{R}^{6}, \delta(x)$ denotes the distance between $x$ and $\mathcal{M}$. In view of the preceding, $x(t)$ will converge to $\mathcal{E}$ provided there is a finite time $t_{1}$ such that $x\left(t_{1}\right) \in \mathcal{S}\left(\rho^{*}\right)$. To prove that such a time must exist, we will assume the contrary and show that this leads to a contradiction.

Suppose that for all $t, x(t)$ is in the complement of $\mathcal{S}\left(\rho^{*}\right)$ which we denote by $\overline{\mathcal{S}}\left(\rho^{*}\right)$. Thus for all $t, x(t)$ is in the closed set $\mathcal{V}=\left\{x: \delta(x) \geq \gamma, x \in \overline{\mathcal{S}}\left(\rho^{*}\right)\right\}$ which in turn is disjoint with $\mathcal{M} \cup \mathcal{E}$. Note that $\mathcal{V}$ contains the closure of the set $\mathcal{T}=\mathcal{X} \cap \mathcal{V}$; this means that $\mathcal{M} \cup \mathcal{E}$ and the closure of $\mathcal{T}$ are disjoint. Thus if we define

$$
\sigma=\sup _{x \in \mathcal{T}} \Phi(x)
$$

then $\sigma<0$ because of Lemma 4. Since $\dot{V}(t)=\Phi(x(t))$, it must be true that $\dot{V}(t) \leq$ $-\sigma, \quad t<\infty$. Thus $V \leq V(0)-\sigma t$ for all $t<\infty$. But this is impossible because $V$ is nonnegative. Therefore $x$ enters $\mathcal{S}\left(\rho^{*}\right)$ in finite time and consequently converges to $\mathcal{E}$.

We now turn to the problem of showing that all trajectories starting outside of $\mathcal{N}$ must be bounded away from $\mathcal{M}$, even in the limit as $t \rightarrow \infty$. As a first step toward this end, let us note that

$$
\operatorname{det}\left[z_{1}(t) \quad z_{2}(t)\right]=e^{-\int_{\tau}^{t}\left(e_{1}(s)+e_{2}(s)+e_{3}(s)\right) d s} \operatorname{det}\left[z_{1}(\tau) \quad z_{2}(\tau)\right] t \geq \tau \geq 0
$$

because of (19). In view of (18) it must therefore be true that any trajectory starting outside of $\mathcal{N}$ cannot enter $\mathcal{N}$ and therefore $\mathcal{M}$ \} in finite time. It remains to be shown that any such trajectory also cannot enter $\mathcal{M}$ even in the limit as $t \rightarrow \infty$. To prove that this is so we need the following facts. Let $\Theta: \mathcal{X} \rightarrow \mathbb{R}$ denote the function $\Theta(x)=e_{1}+e_{2}+e_{3}$ with the $z_{i}$ and $e_{i}$ as defined previously. Obviously $\Theta(x(t))=e_{1}(t)+e_{2}(t)+e_{3}(t)$ when $x(t)$ is a solution to the overall system and the 
$e_{i}(t)$ are the values of the error signals along that solution. We are interested in the following property of $\Theta$ when viewed as a function on $\mathcal{X}$.

LEMMA 5.

$$
\lim _{x \rightarrow \mathcal{M}} \Theta(x)<0
$$

Proof. We first prove that $\Theta(x)$ eventually becomes negative as $x \rightarrow \mathcal{M}_{0}$. Since the $e_{i}$ are continuous on $\mathcal{X}$, it is enough to show $e_{1}+e_{2}+e_{3}<0$ for all values of $x \in \mathcal{M}_{0}$. Since $\mathcal{M}_{0}$ is a subset of $\mathcal{N}$, it is always true on $\mathcal{M}_{0}$ that $\left\|z_{i}\right\|=\left\|z_{[i]}\right\|+\left\|z_{[i]]}\right\|$ for some $i \in\{1,2,3\}$. Without loss of generality, suppose that at some point $x \in \mathcal{M}_{0}$, $\left\|z_{1}\right\|=\left\|z_{2}\right\|+\left\|z_{3}\right\|$. This implies that $\left\|z_{1}\right\|>\left\|z_{2}\right\|$ because $z_{3} \neq 0$. Observe that if $e_{i}=0$ for some $i \in\{1,2,3\}$, then $e_{i}=0$ for all $i \in\{1,2,3\}$ because $z_{1} e_{1}=z_{2} e_{2}=z_{3} e_{3}$ and $\left\|z_{1}\right\|,\left\|z_{2}\right\|,\left\|z_{3}\right\|>0$. However, $e_{1}, e_{2}$ and $e_{3}$ cannot all be zero because $\mathcal{M}_{0}$ and $\mathcal{E}$ are disjoint. Thus $e_{i} \neq 0$ for all $i \in\{1,2,3\}$. Since $e_{1} z_{1}=e_{2} z_{2}$ and $\left\|z_{1}\right\|>\left\|z_{2}\right\|$, it follows that $\left|e_{1}\right|<\left|e_{2}\right|$. Because of the equality $\left\|z_{1}\right\|=\left\|z_{2}\right\|+\left\|z_{3}\right\|$, we know that the sign of $z_{1}$ is the negative of the signs of both $z_{2}$ and $z_{3}$; this implies that $e_{1} e_{2}<0$ and $e_{1} e_{3}<0$. Now suppose $e_{1}<0$. Then $e_{2}>0$ and $e_{3}>0$; therefore $\left\|z_{1}\right\|<d_{1}$, $\left\|z_{2}\right\|>d_{2}$ and $\left\|z_{3}\right\|>d_{3}$. Consequently $d_{1}>\left\|z_{1}\right\|=\left\|z_{2}\right\|+\left\|z_{3}\right\|>d_{2}+d_{3}$ which contradicts the triangle inequality $d_{1}<d_{2}+d_{3}$. Hence, it must be true that $e_{1}>0$, $e_{2}<0$ and $e_{3}<0$. In view of the fact $\left|e_{1}\right|<\left|e_{2}\right|$, we conclude that $e_{1}+e_{2}+e_{3}<e_{3}<0$.

Now we prove if $x$ approaches $\mathcal{M}_{i}, i=1,2,3$, then $\Theta(x)<0$. Suppose $x \rightarrow \mathcal{M}_{1}$. Then $z_{1} \rightarrow 0$ and $z_{2} e_{2}-z_{3} e_{3} \rightarrow 0$. The former implies that $z_{2}+z_{3} \rightarrow 0$ because of (13). Two things can happen: Either $z_{2}$ and \{therefore $z_{3}$ approach zero or both are bounded away from 0 . If the former is true, $e_{1}$ and $e_{2}$ eventually both become negative because of their definitions. On the other hand, if $z_{2}$ and $z_{3}$ are both bounded away from zero, then $e_{2}+e_{3} \rightarrow 0$ because both $z_{2} e_{2}-z_{3} e_{3}$ and $z_{2}+z_{3}$ approach zero. In summary, the sum $e_{2}+e_{3}$ must eventually either become negative or at worst, arbitrarily small in magnitude as $x$ approaches $\mathcal{M}_{1}$. Meanwhile, $e_{1}$ must eventually become negative as $x$ approaches $\mathcal{M}_{1}$ because of its definition and the fact that $z_{1} \rightarrow 0$. These observations imply that under all conditions, the sum $e_{1}+e_{2}+e_{3}$ must eventually become negative as $x$ approaches $\mathcal{M}_{1}$. Using the same reasoning, one reaches the same conclusion if $x$ approaches either $\mathcal{M}_{2}$ or $\mathcal{M}_{3}$.

Finally, we prove that $\Theta(x)<0$ when $x \rightarrow \mathcal{Z}_{0}$. Since in this case $z_{1}, z_{2}$ and $z_{3}$ all tend to zero, the $e_{1}, e_{2}$, and $e_{3}$ all tend to negative values because of their definitions. Therefore the sum $e_{1}+e_{2}+e_{3}$ has a negative limit in this case too. This completes the proof.

We are now ready to show that any trajectory starting outside of $\mathcal{N}$, cannot approach $\mathcal{M}$ in the limit as $t \rightarrow \infty$. Suppose the opposite is true, namely that $x(t)$ is a trajectory starting outside of $\mathcal{N}$ which approaches $\mathcal{M}$ as $t \rightarrow \infty$. Then in view of 
(34), (18), and the fact that $\mathcal{M} \subset \mathcal{N}$,

$$
\lim _{t \rightarrow \infty}\left|\operatorname{det}\left[\begin{array}{ll}
z_{1} & z_{2}
\end{array}\right]\right|=0
$$

We will now show that this is false.

In view of Lemma 5 , there must be an open set $\mathcal{V}$ containing $\mathcal{M}$ on which the inequality in the lemma continues to hold. In view of Lemma 2 and the fact that $\mathcal{M} \subset \mathcal{N}$, it is possible to choose $\mathcal{V}$ small enough so that in addition to the preceding, $\mathcal{V}$ and $\mathcal{E}$ are disjoint. For $x(t)$ to approach $\mathcal{M}$ means that for some finite time $T$, $x(t) \in \mathcal{V}, t \in[T, \infty)$. This implies that $e_{1}+e_{2}+e_{3}<0, t \geq T$. In view of (34), $\left|\operatorname{det}\left[\begin{array}{ll}z_{1} & z_{2}\end{array}\right]\right| \geq\left|\operatorname{det}\left[z_{1}(T) z_{2}(T)\right]\right|, t \geq T$. But

$$
\left|\operatorname{det}\left[z_{1}(T) \quad z_{2}(T)\right]\right|=e^{-\int_{0}^{T}\left(e_{1}(s)+e_{2}(s)+e_{3}(s)\right) d s}\left|\operatorname{det}\left[z_{1}(0) \quad z_{2}(0)\right]\right|
$$

Moreover, $\left|\operatorname{det}\left[z_{1}(0) \quad z_{2}(0)\right]\right|>0$ because $z$ starts outside of $\mathcal{N}$. Therefore $\left|\operatorname{det}\left[\begin{array}{ll}z_{1} & z_{2}\end{array}\right]\right|$ $>\left|\operatorname{det}\left[z_{1}(T) z_{2}(T)\right]\right|>0, t \geq T$ which contradicts (36). This completes the proof of Theorem 1.

The preceding proves among other things that trajectories starting outside of $\mathcal{N}$ cannot approach $\mathcal{M}$. But $\mathcal{N} \cap \mathcal{X}$ is an invariant manifold. Moreover, we've already proved that all trajectories starting outside of $\mathcal{N}$ converge to $\mathcal{E}$. We can therefore conclude that any trajectory starting outside of $\mathcal{N}$ can never enter $\mathcal{N}$.

4. Concluding Remarks. The aim of this paper has been to analyze the control laws proposed in [5], [4] and [3] using a single approach. The convergence of $x$ trajectories to $\mathcal{E}$ from points outside of $\mathcal{N}$ could also be deduced from the Lasalle Invariance Principle $\{[7]\}$ by defining the domain of definition of the original system to be $\mathbb{R}^{6}-\mathcal{N}$. However to make use of this principle one would still have to prove that trajectories starting at points outside of $\mathcal{N}$ are bounded and bounded away from $\mathcal{M}$, since the pre-compactness hypothesis of the principle demands this. Indeed, this is roughly the approach taken in [3]. An advantage of the approach taken in this paper is that it enables one to establish exponential convergence whereas, without further elaboration, the Lasalle Invarance Principle only provides asymptotic convergence.

It is likely that findings similar to those deduced in this paper can be shown to hold for any given rigid formation in the plane consisting of any number of autonomous agents, provided each agent admits a kinematic point model as assumed in this paper, and the distance constraints which each agent must satisfy are consistent [8]. This is suggested not only by the results derived here but also by the findings of [9] which address the distance constrained formation maintenance problem assuming small errors in agent positions.

Despite the preceding optimism, advances in the spirit of this paper which go beyond single cycle formations have so far proved to be especially difficult to obtain. For example, it is not yet clear how to control the two-cycle directed formation 


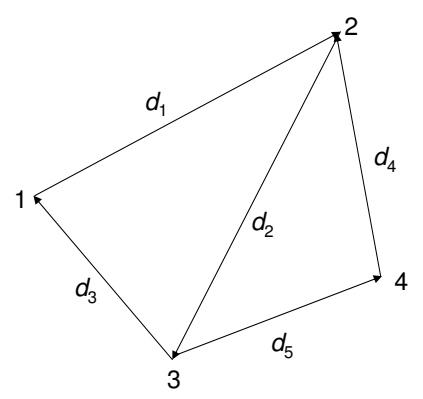

FIG. 2. A Two-Cycle, Directed Point Formation

with provably correct controls, unless one is willing to accept results of a local nature. One feature of this particular formation which distinguishes it from the triangle formation in Figure 1, is that in the two-cycle case one agent, namely agent 3, has two co-leaders whereas each agent in the triangle formation has only one. Understanding how to deal with two co-leaders is probably the key to advancing the findings of this paper.

Another direction in which formation control research might proceed, would be to study the problem assuming more realistic kinematic and dynamic agent models. One possible way to do this, while taking advantage point model results such as those in this paper, might be to consider the use of virtual shells [10]. In relation to a triangular formation and perhaps generally, one could also envisage that replacement of a single integrator agent model by a double integrator model would allow both distance-based shape control and convergence of agent velocities to a common velocity, perhaps that of a designated leader.

\section{REFERENCES}

[1] M. Cao, C. Yu, A.S. Morse, B. D. O. Anderson, and S. Dasgupta. Generalized controller for directed triangle formations. In: Proceedings of the 2008 IFAC Congress, pages 65906595, 2008.

[2] J. BAILlieul AND A. SURI. Information patterns and hedging Brockett's theorem in controlling vehicle formations. In: Proc. of the 42th IEEE Conference on Decision and Control, pages $194-203,2003$.

[3] S. L. Smith, M. E. Broucke, And B. A. Francis. a multi-agent system to an equilibrium polygon formation. In: Proc. of the MTNS, pages 2415-2424, 2006.

[4] B. D. O. Anderson, C. Yu, S. Dasgupta, and A. S. Morse. Control of a three-coleader formation in the plane. Systems and Control Letters, 56:573-578, 2007.

[5] M. CaO, A.S. Morse, C. Yu, B. D. O. Anderson, And S. Dasgupta. Controlling a triangular formation of mobile autonomous agents. In: Proceedings of the 46th IEEE Conference on Decision and Control, pages 3603-3608, 2007.

[6] T. Eren, P. N. Belhumeur, B. D. O. Anderson, And A. S. Morse. A framework for maintaining formations based on rigidity. In: Proceedings of the 2002 IFAC Congress, 
pages $2752-2757,2002$.

[7] J. P. Lasalle. The Stability of Dynamical Systems. Society for Industrial and Applied Mathematics, 1976.

[8] J. M. Hendrickx, B. D. O. Anderson, J.-C. Delvenne, And V. D. Blondel. Directed graphs for the analysis of rigidity and persistence in autonomous agent systems. Int. J. Robust Nonlinear Control, 17(2007), pp. 960-981.

[9] C. Yu, B. D. O. Anderson, S. Dasgupta, and B. Fidan. Control of minimally persistent formations in the plane. SIAM J Control and Optimization, 48:1(2009), pp. 206-233.

[10] A. S. MorSE. Virtual shells for avoiding collisions. Presentation available on line at the URL http://entity.eng.yale.edu/controls/publications.html, jul 2001. 